\title{
Full Shoe Print Recognition based on Pass Band DCT and Partial Shoe Print Identification using Overlapped Block Method for Degraded Images
}

\author{
S. Rathinavel \\ Research scholar, Department of ECE \\ Excel Engineering College, Anna University \\ Chennai, India
}

\author{
S.Arumugam \\ Chief Executive Officer, Department of ECE \\ Nandha Engineering College, Anna University \\ Chennai, India
}

\begin{abstract}
In this paper, a novel approach is made to discard the degradations in full shoe prints and partial shoe prints, in processing those images for recognition. A pass band DCT coefficient has been used to extract feature vectors. A more robust approach has been dealt with to find the matching between the partial shoe prints and the images in the data base. This method makes the shoe print recognition process more robust against degradations like noises, orientations and blurred images which are common in shoe print images and also helps in saving the processing time and memory consumption.
\end{abstract}

\section{General Terms}

Forensic analysis, Pattern Recognition and Artificial Intelligence

\section{Keywords}

Shoe print, Fisher Linear Discriminant (FLD), recognition, forensic science, Principal Component Analysis (PCA).

\section{INTRODUCTION}

In forensic analysis, shoe marks provide valuable information as evidence against accused persons. In many cases, shoe prints can be positively identified as having been made by a specific shoe to the exclusion of all other shoes. The identification is based on the physical match of individual designs. The evidence provided by a positively identified shoe mark is as strong as the evidence from fingerprints, tool marks, and typewritten impressions [1]. But this matching alone may not be the only criteria to identify the suspected person in crime scene. But, still this information is a valuable one. Due to the infinite designs available in the market, with distinctive outsole patterns, this implies that there exists specific general population of shoe prints [1]. If the model of a shoe can be determined from its mark, then this can abruptly narrow the search for a particular suspect. So, at least the forensic department can narrow down their search of suspected persons. The data base formation is the first step in shoe print processing. So matching algorithm starts with image acquisition process. An image of a shoe print is first obtained using a technique such as photography, electrostatic lifting or by making a cast when the impression is in soil, snow or sand. Further, in the forensic laboratories, the image of the shoe mark is compared with the shoeprints and shoe impressions of known shoe samples. A process of detection and recovery of footwear impression evidence and of comparison of the impressions with suspect shoes has been explained in [1]. The photograph of the impression or of the lifted impression or cast can be subsequently scanned and a digital image is produced. Forensic analysis requires comparison of this image against specific databases. These databases include, database of prints made by shoes of already existing criminals and previously available on the market and database of shoe prints found at other crime scenes. Comparing crime scene shoe mark images to databases is currently a difficult task and it is usually conducted manually by searching paper catalogues or computer databases. Our task is to simplify the matching process against degradations and help the forensic scientists for faster search.

\section{EXISITING WORK}

Matching of shoe prints by several methods has been reported early [2-9]. However, several practical difficulties exist in the classification of shoeprints-both manual and automated.

Statistical techniques have been widely used for shoe recognition and in shoe print analysis to extract the matched image from the data base. Dimensionality reduction is essential for extracting effective features and reducing computational complexity in classification stage. Principal component analysis (PCA) [12], [13], Linear discriminate analysis (LDA) [14] and Discrete cosine transform (DCT) [15] are the main techniques used for data reduction and feature extraction in the appearance based approaches. DCT, Eigen faces [12] and Fisher faces [16] have been proved to be very successful among the others. All these methods that extract features to optimally represent test shoe image belong to a class and separate the shoe prints. In the literature it has been found that most efforts are given mainly on developing feature extraction methods and employing powerful classifiers such as Euclidean distance Classifier, Hidden Markov Models (HMMs) [17] and support vector machine (SVM). In this work, it is proposed to reduce the dimensions, in the initial stage itself, which gives two advantages, one being the reduced memory requirement and the other being the robust against degradations such as noise and lighting conditions. The full shoe print image using DCT is shown in section 3. Partial Shoe print processing is given in section 4 . The rest of the paper deals with results and performance analysis.

\section{SYSTEM FOR FULL SHOE PRINT}

In this paper we propose an integrated technique for a full shoe print recognition system based on the DCT components analysis in FLD with PCA and partial shoe print recognition using overlapping block method. The following steps are involved in this preprocessing of a full shoe print image. 


\subsection{Resizing}

The captured images may be in different sizes. It depends on the place where the image has been captured. So before processing the image, it should be converted into a particular size. Here, the images are made to standard size as $400 \times 300$.

\subsection{Normalization}

Normalization is used to standardize the intensity values in an image by adjusting the range of grey-level values so that it lies within a desired range of values. Normalization is pixel-wise operation. The main purpose of normalization is to reduce the variation in gray level values, and to facilitate the subsequent processing steps.

\subsection{Orientation estimation}

The orientation image represents an intrinsic property of the shoeprint images. In this work an automatic orientation correction is proposed before the images are trained. The shoe (bottom sole) image in horizontal view, is first processed to find the binary image using a fixed threshold. This is followed by detecting the contour and then by finding the extreme end points. The angle joining this line $\mathrm{AB}$ is the tilt angle. So based on this angle rotation, correction is performed using Radon Transforms (Figure 1 and Figure 2).

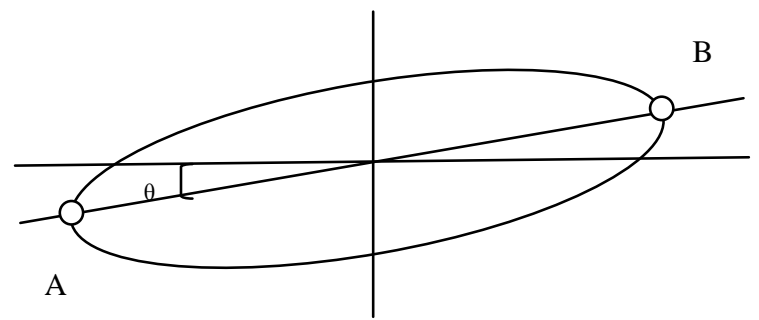

Figure 1. Before orientation correction

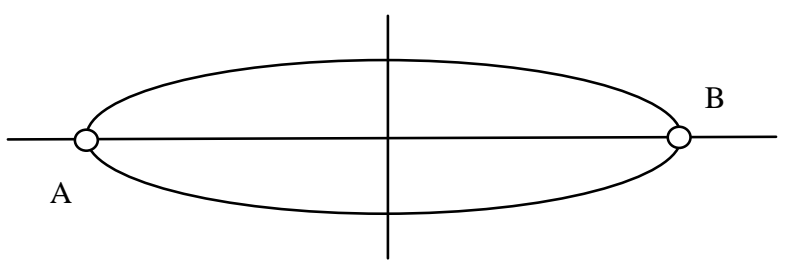

Figure 2. After orientation correction

\subsection{Discrete Cosine Transform}

A discrete cosine transform (DCT) expresses a sequence of finitely many data points in terms of a sum of cosine functions oscillating at different frequencies. The DCT is conceptually similar to the discrete Fourier transform(DFT), except that the DCT does a better job of concentrating energy into lower order coefficients than does the DFT for image data also the DCT is purely real (only magnitude).

A DCT operation on a block of pixels produces coefficients that are similar to the frequency domain coefficients produced by a DFT operation. An N-point DCT has the same frequency resolution as and is closely related to a $2 \mathrm{~N}$-point DFT. The $\mathrm{N}$ frequencies of a $2 \mathrm{~N}$ point DFT correspond to $\mathrm{N}$ points on the upper half of the unit circle in the complex frequency plane. For most images, after transformation the majority of signal energy is carried by just a few of the low order DCT coefficients. These coefficients can be more finely quantized than the higher order coefficients. Many higher order coefficients may be quantized to 0 (this allows for very efficient run-level coding).

The input image is divided into several horizontal and vertical blocks, with each block sizing $8 \times 8$. So, in this case, $N$ is typically 8 and the DCT is applied to each row and column of the block. The result is an $8 \times 8$ transform coefficient array in which the $(0,0)$ element (top-left) is the DC (zero-frequency) component and entries with increasing vertical and horizontal index values represent higher vertical and horizontal spatial frequencies. With reference to [18], the DCT coefficients are reordered by the zigzag scan. This scan method reorders all the DCT coefficients from low frequency to high frequency. For most images, it is equivalent to sorting according to importance, since the perturbation in the low frequency components is generally more perceivable to human eyes than high frequency components.

Zigzag scanning process is performed on DCT coefficients as shown in Figure.3. After zigzag scanning, (1x64) vector is produced. This vector contains coefficients of DCT block from low frequency to high frequency. Low-pass, Band-pass, Highpass filters are applied to this vector for constructing a feature vector about whole spectrum range of the sub block. After this, FLD and PCA is performed on the DCT coefficients.

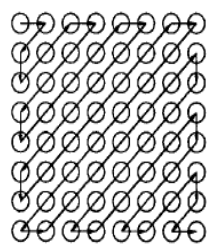

Figure 3. 8x8 matrix DCT coefficients

\subsection{Fisher Linear Discriminant}

1. To Compute the transformation matrix of $\mathrm{K}-\mathrm{L}$ transformation $\mathrm{U}_{\mathrm{KL}}$ :

$$
U_{K L}=\arg \max \left|U^{T} S_{T} U\right|=\left[u_{1}, u_{2}, \ldots u_{n}\right]
$$

where $\left\{u_{\mathrm{i}}=1,2, \ldots . \mathrm{n}\right\}$ is the set of eigenvector of $\mathrm{S}_{\mathrm{T}}$ corresponding to the nonzero Eigen value and $S_{T}$ is the total scatter matrix defined as

$$
S_{T}=\sum_{k=1}^{N}\left(x_{k}-\mu\right)\left(x_{k}-\mu\right)^{T}
$$

2. Compute the transformed within-class scatter matrix $S^{\prime}{ }_{w}$, which is a full rank matrix:

$$
S_{w}^{\prime}=U_{K L}^{T} S_{w} U_{K L}
$$

3. Compute the transformed between-class scatter matrix $\mathrm{S}_{\mathrm{B}}$ :

$$
S_{B}^{\prime}=U_{K L}^{T} S_{B} U_{K L}
$$

4. The standard FLD defined is used to the transformed samples to obtain $\mathrm{W}_{\text {fld }}$ : 


$$
\begin{aligned}
& W_{f l d}={ }_{w}^{\arg \max } \frac{\left|W^{T} S_{B}^{\prime} W\right|}{\left|W^{T} S_{w}^{\prime} W\right|} \\
& =\arg \max _{w} \frac{\left|W^{T} U_{K L}^{T} S_{B} U_{K L} W\right|}{\left|W^{T} U_{K L}^{T} S_{w} U_{K L} W\right|}
\end{aligned}
$$

Compute $\mathrm{W}_{\text {opt }}$ :

$$
W_{o p t}^{T}=W_{f l d}^{T} U_{\mathrm{KL}}^{T}
$$

Fisher's linear discriminant is a classification method that projects high-dimensional data onto a line and performs classification in this one-dimensional space. The projection maximizes the distance between the means of the two classes while minimizing the variance within each class. This defines the Fisher criterion, which is maximized over all linear projections, $w$ :

$$
J(w)=\frac{\left|m_{1}-m_{2}\right|^{2}}{S_{1}^{2}+S_{2}^{2}}
$$

where $m$ represents a mean, $s^{2}$ represents a variance, and the subscripts denote the two classes. In signal theory, this criterion is also known as the signal-to-interference ratio. Maximizing this criterion yields a closed form solution that involves the inverse of a covariance-like matrix. This method has strong parallels to linear perceptrons. We learn the threshold by optimizing a cost function on the training set. Fisher's linear discriminant is the first step on the way to discriminant Factor Analysis. It can be generalized to more than just 2 classes. If the data sits in a $n$ dimensional space and there are $p$ classes with $p<n$, then $(p-1)$ orthogonal 1-D projection lines may be defined. The first one is just the generalized Fisher's linear discriminant. The second one is defined as sitting in the $(n-1)$ dimensional subspace orthogonal to it, and maximizing the generalized Fisher's criterion for the classes once projected in that subspace. These are the discriminant factors.

\subsection{Principal Component Analysis}

PCA also known as Karhunen-Loeve method is a technique commonly used for dimensionality reduction in computer vision, particularly in face recognition. A method called Eigenface, based on PCA was used in face recognition in [12]. In our proposed work, the PCA, the principal components of the distribution of shoe prints or the eigenvectors of the covariance matrix of the set of shoe print images are sought treating an image as a point in a very high dimensional space. These eigenvectors can be thought of as a set of features that together characterize the variation between shoe print images. Each image contributes to each eigenvector so that a sort of ghostly shoe print called eigenshoe can be formed. These features vectors from FLD and PCA are now extracted during the training phase. Later a test image is selected, whose target image stored in the test data base is also run through this same algorithm and feature vectors are extracted. Euclidean distance is calculated between this test feature vector and all the feature vectors of train data base obtained during training phase. The index of the minimum distance, is taken as the most relevant image and shown as matched image.

\section{SYSTEM FOR PARTIAL SHOE PRINT}

In actual scenario, partial shoeprints are much more common at crime scenes than full shoeprints and can provide valuable forensic evidence. Hence, the performance of a system in processing partial images of shoeprints becomes valid interest. Partial Shoe prints may be of two categories (i) Acquired partial shoe print and (ii) Generated partial shoe print. We generated partial shoe prints as follows.

\subsection{Generation of Partial-Prints}

Partial print images were generated from each full-print image. They were generated in conventional methods as follows: They were divided into four sub images. So template input was in the order of $25 \%$ or little lesser than that. So it is worth to enhance the matching process, using FFT using the phase information. In our work, we use $5 \%$ to $10 \%$ of the total area of the full shoe print images. This performance is achieved by means of block overlapping method as given in the Figure 4. The matching performance was carried out for 235 full shoe print images. A query images were given as input with added additive white Gaussian noise with mean zero and variance 0.01 and matched image is obtained.

\subsection{Overlapped Block Method}

Fourier Transforms (FT) have been used for classification of partial prints $[10,11]$. The FT is invariant to translation and rotation and this approach shows that although footwear impressions are processed globally, they are encoded in terms of the local information evident in them. In our proposed work, overlapped blocks have been used as shown in the Figure 4. Though this increases the fuzziness in the system, it gives better performance.

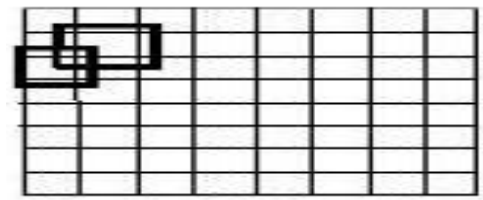

Figure 4. 8x8 matrix DCT coefficients

\section{OUTPUTS}

The outputs have been shown in GUI window as shown in Figure 5 to Figure 8, developed with MATLAB image processing tool box. The experiment had been conducted with a data base of 235 shoe print images. Initially the test image was corrupted by Gaussian noise with mean 0 and Variance 0.01 and filtered with median filter. The results obtained are as follows.

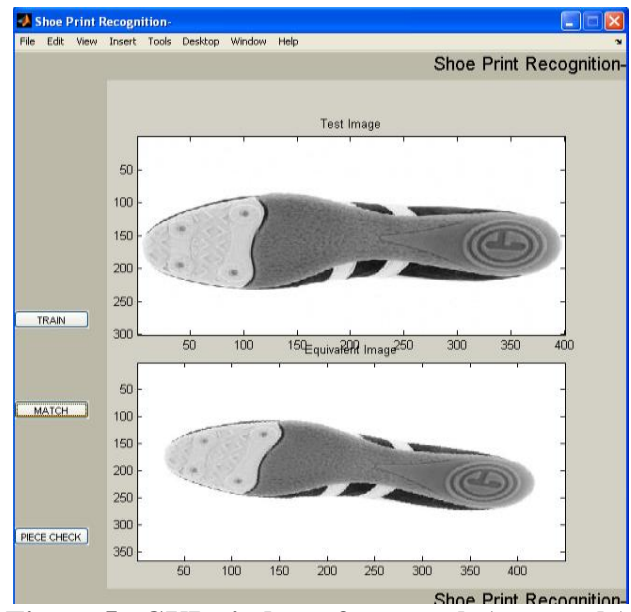

Figure 5. GUI window after match (+ve angle) 


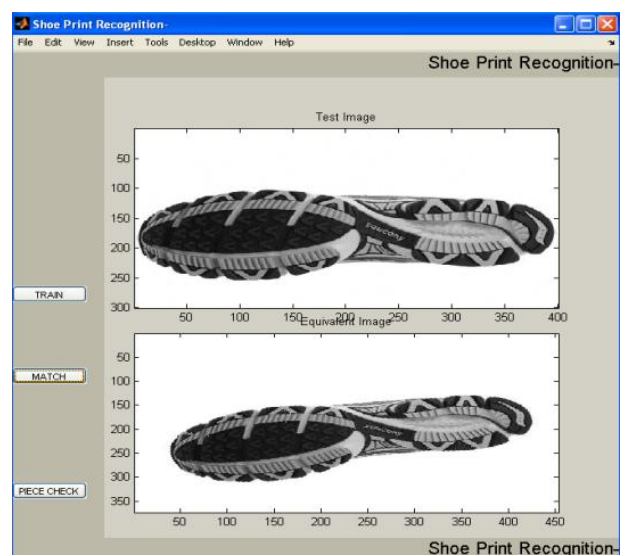

Figure 6. GUI window after match (-ve angle)

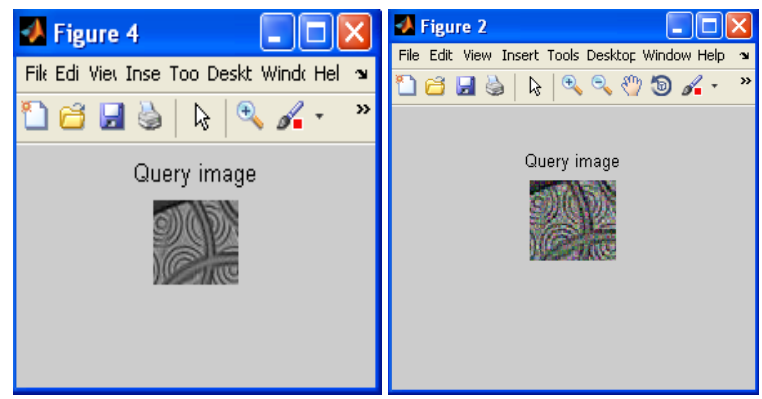

Figure 7 Query image with and without noise

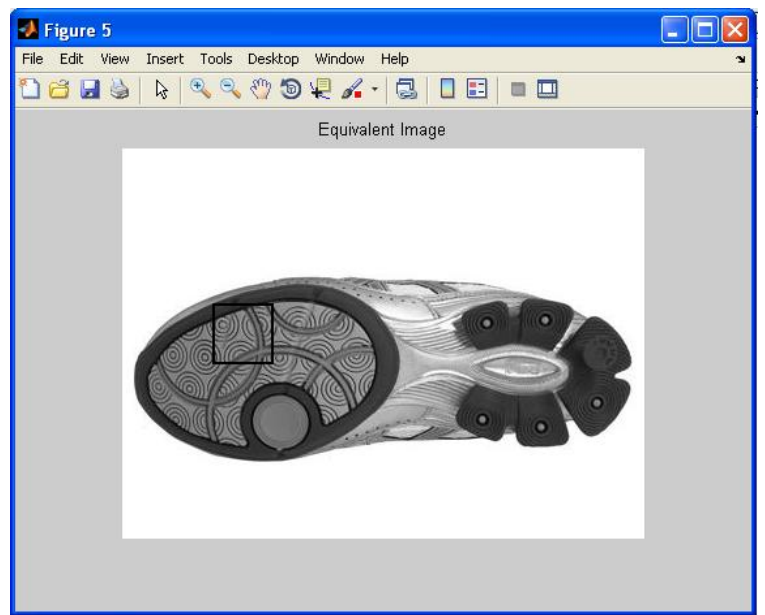

Figure 8 Matched portion of the Shoe Sole bottom

\section{PERFORMANCE ANALYSIS}

From the obtained results as shown in Figure 9, it is seen that as the noise variance increases, the Euclidean distance of the target image also increases for a fixed 48 number of DCT coefficients in an $8 \times 8$ matrix. This implies that the algorithm is immune to noise, if the noise variance is in the order of 0.01 . More over by varying the number of DCT coefficients in an $8 \times 8$ matrix, it is proved that only with one coefficient it is possible to recognize the target image, as it is evident with the least value of Euclidean distance of 0.00 when a match with noiseless input is attempted. Moreover, the performance without noise is optimum when the coefficients are taken around 32 as it is clear from Figure 10. So, it is shown that the dimensionality reduction has been achieved. This is very useful in ASIC implementation, as this reduces the computation time, which is because we take only few number of DCT coefficients], instead of taking the whole 64 coefficients. Also in EPROM, it is enough to store only those few coefficients, which reduces the total memory consumed. Though there exists so many works, to the best of our knowledge, the works concentrate only on efficient recognition rather than the memory consumed for the process. As it is necessary to implement a prototype model, it is mandatory to reduce the processing time and the memory consumed during the process.

Table 1. Noise Variance Vs Euclidean Distance

\begin{tabular}{|c|c|c|}
\hline S.No & $\begin{array}{c}\text { Noise variance } \\
\text { (DCT coefficients) }\end{array}$ & $\begin{array}{c}\text { Euclidean distance of } \\
\text { target image }\end{array}$ \\
\hline 1 & 0.01 & 8.6 \\
\hline 2 & 0.02 & 22.1 \\
\hline 3 & 0.03 & 37.5 \\
\hline 4 & 0.04 & 53 \\
\hline 5 & 0.05 & 174 \\
\hline
\end{tabular}

Table 2. DCT Coefficients Vs Euclidean Distance

\begin{tabular}{|c|c|c|}
\hline S.No & $\begin{array}{c}\text { No of DCT } \\
\text { coefficients }\end{array}$ & $\begin{array}{c}\text { Euclidean distance of } \\
\text { target image } \boldsymbol{\gamma}=\mathbf{0}\end{array}$ \\
\hline 1 & 4 & 5.6 \\
\hline 2 & 8 & 0.3 \\
\hline 3 & 16 & 0.1 \\
\hline 4 & 32 & 0 \\
\hline 5 & 48 & 0 \\
\hline
\end{tabular}

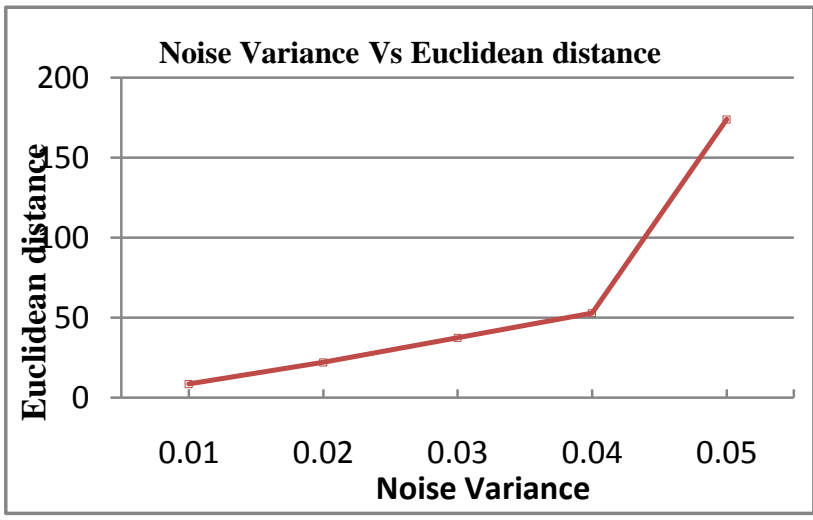

Figure 9 Noise Variance Vs Euclidean distance 


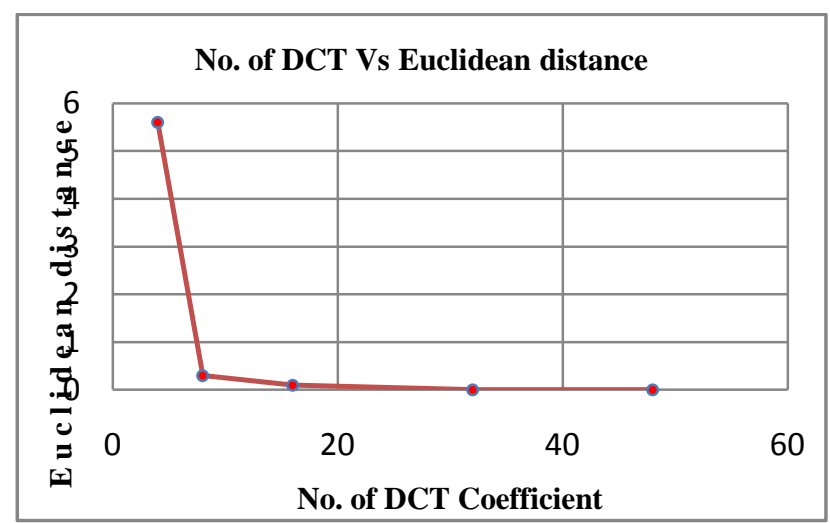

Figure 10. No. of DCT Coefficients Vs Euclidean distance

Keeping this as a primary issue it is suggested to reduce the dimensions of the feature vectors extracted in recognition process. In conventional systems, with a full shoe print image size of $400 x 300$, the total memory consumption is $120 \mathrm{k}$ bytes which acts as a buffer from where the FLD based feature vectors are extracted. In our proposed work, instead of image pixels, DCT coefficients $[18,19]$ are applied as input to FLD process. As we do selection process in zigzag scan, it results in reduced dimensions, which is just $60 \mathrm{k}$ bytes, (if the number of DCT coefficients taken is 32 ). In addition to this, PCA also reduces the dimension of the feature vectors which is an additional advantage. In this work it is proved that the memory requirement is less for the real time hardware implementation, without any compromise in the recognition by feature vectors. Hence it is evident that both computation time and the memory requirement is reduced. There is a saving of 0.4 seconds in recognition time. As we introduce, DCT coefficient choosing process in this work, it is transparent that, the algorithm running time increases, but even then we are able to save recognition time. More over, the main advantage is that the memory consumption is reduced approximately by 16 times, any how this depends on the noise level in the input query image.

\section{CONCLUSION}

In this work, a new shoe print recognition work had been done with dimensionality reduction with pass band DCT and FLD combined with PCA. Hence it is claimed that, many advantages are enjoyed as mentioned in the previous section. All other conventional systems do not focus on the memory constraint. Though those works focus on speed of computations, it makes use of the full dimension of the actual image or their coefficients. In our work, efforts have been taken to solve this memory constraint. By reducing the number of DCT coefficients, to be considered for FLD and PCA based feature extraction. Also partial shoe prints have been taken into account and the matching had been performed using phase information under noisy environment..

\section{ACKNOWLEDGMENTS}

Our thanks to the institution for supporting us with Simulation software.

\section{REFERENCES}

[1] W. Bodziak, Footwear Impression Evidence Detection, Recovery and Examination, 2000, Second ed. CRC Press.

[2] A. Girod, 1996, Computer classification of the shoeprint of burglars' shoes, Forensic Science Int. Vol 82, pp 59-65..

[3] M. Phillips, Shoeprint image coding and retrieval system, 1995, Proceedings of the European Convention on Security and Detection, pp. 267-271.

[4] N. Sawyer, "SHOE-FIT: A computerized shoe print database," 1995, Proc. European Convention on Security and Detection, pp 86-89.

[5] W.Ashley, What shoe was that? the use of computerized image database to assist in identification, Forensic Science Int. 82(1) (1996) 7-20.

[6] S. Mikkonen, T. Astikainenn, Database classification system for shoe sole patterns-identification of partial footwear impression found at a scene of crime, 1994, Journal of Forensic Science 39(5), pp 1227- 1236.

[7] Z. Geradts, J. Keijzer, The image-database REBEZO for shoeprints with developments on automatic classification of shoe outsole designs, 1996, Forensic Science Int. 82, pp 21-31.

[8] A. Alexander, A. Bouridane, D.Crookes, Automatic classification and recognition of shoeprints, 1999, Seventh International IEEE Conference on Image Processing and Its Applications Vol 2, pp 638-641.

[9] A. Bouridane, A. Alexander, M. Nibouche, D. Crookes, "Application of fractals to the detection and classification of shoeprints," 2000, IEEE Conference, 0-7803-6297, pp 474-477.

[10] C. Huynh, P. de Chazal, D. McErlean, R. Reilly, T.Hannigan, L.Fleud, Automatic classification of shoeprints for use in forensic science based on the fourier transform, Proc. International Conference on Image Processing 3 , pp 569-572.

[11] P. de Chazal, J. Flynn, R. B. Reilly, Automated processing of shoeprint images based on the fourier transform for use in forensic science, Pattern Analysis and Machine Intelligence, IEEE Transactions on 27 (2005) 341-350.

[12] Matthew Turk and Alex Pentland, "Eigenfaces for Recognition". Journal of Cognitive Neuroscience, Vol.3, No.1, pp. 71-86, 1991.

[13] P.N.Belhumeur, J.P.Hespanha and D.J. Kriegman, "Eigenfaces versus fisherfaces: Recognition using class specific Linear projection," IEEE Trans. Pattern Anal. Mach. Intell., Vol.19, No.7, pp. 711-720, Jul.1997.

[14] M. Kirby and L. Sirvoich, "Application of the karhunenLoeve Procedure for the characterization of human faces, "IEEE Trans. Pattern Anal. Mach. Intell., Vol.12, No.1, pp. 103-108, Jan.1990. 
[15] Z.M. Hafed and M.D. Levine, "Face recognition using he discrete cosine transform," Int. J. Comput. Vis., Vol.43, No.3, pp. 167-188,2001.

[16] J. Lu, K.N. Plataniotis, A.N.Venetsanopoulos, "Face recognition using LDA based algorithms, 2003, IEEE Transactions on Neural Networks 14(1), pp.195-200.

[17] H.Othman and T.Aboulnasr, "A separable low complexity 2D HMM with application to face recognition", 2003, IEEE Trans. Pattern Anal. Machine Intell., Vol 25, No.10, pp.1229-1238.
[18] Xiao-Yuan Jing and David Zhang , "A Face and Palmprint Recognition Approach Based on Discriminant DCT Feature Extraction," 2004, IEEE Transactions on Systems, Man, and Cybernetics-Part B: Cybernetics, vol. 34, no. 6, December 2004

[19] Peng-Fei Yu, Dan Xu , "Palmprint Recognition Based On Modified DCT Features and RBF Neural Network," 2008, Proceedings of the Seventh International Conference on Machine Learning and Cybernetics, Kunming, 12-15 July 2008. 